Particle Accelerators, 1990, Vol. 34, pp. 13-23

Reprints available directly from the publisher

Photocopying permitted by license only

(C) 1990 Gordon and Breach, Science Publishers, Inc.

Printed in the United States of America

\title{
RING MAGNETS IN MIGMA SYSTEMS
}

\author{
JOHN P. BLEWETT \\ 310 W. 106th St., New York, N.Y. 10025
}

(Received October 22, 1988)

\section{INTRODUCTION}

The papers of B. Maglich and his associates ${ }^{1-4}$ propose production and use of a charge distribution which they call a "migma" in which charged particles can be collided for nuclear or particle physics, for nuclear fusion and for other purposes.

The charge distribution would be set up in a paraxial magnetic field with cylindrical symmetry, decreasing slowly with increasing distance from the axis of symmetry. In this field, charged particles will travel on orbits passing through the axis and precessing around the axis. These orbits will intersect on or near the axis, where their density will be very high. Hence charged particle collisions can be produced in profusion throughout the central region of the migma.

For use at very high energies where the magnetic field region must become very large, the author has proposed ${ }^{5}$ the use of a ring of magnetic field with low or zero field throughout the central region.

With the original migma field pattern, paraxial forces are weakly restoring ("focusing") everywhere toward the median plane. In the ring field, the inner part of the field pattern (where the field is increasing with radius) will be axially defocusing, while the outer part will be axially focusing. In combination, as in alternating-gradient focusing systems, these fields will provide a net focusing effect if their configuration meets certain criteria, as presented below.

The analysis presented here is approximate, but will indicate acceptable ranges of important parameters. Actual design of a ring magnet system will call for detailed computer study of orbits and magnetic field configurations.

The field pattern considered here will have the form shown in Fig. 1:

From point $\mathrm{O}$ to point $\mathrm{A}$, the paraxial magnetic field $B_{z}$ is 0 .

From point $\mathrm{A}$ to point $\mathrm{B}, B_{z}$ increases linearly from 0 to $B_{0}$.

From point $\mathrm{B}$ to point $\mathrm{C}, B_{z}$ decreases linearly at a rate to be determined.

It will be assumed that orbits do not travel past point $\mathrm{C}$, so the shape of the field for radii greater than the distance $\mathrm{OC}$ is immaterial.

It will be assumed also that the distances $\mathrm{AB}$ and $\mathrm{BC}$ are small compared with OA. 


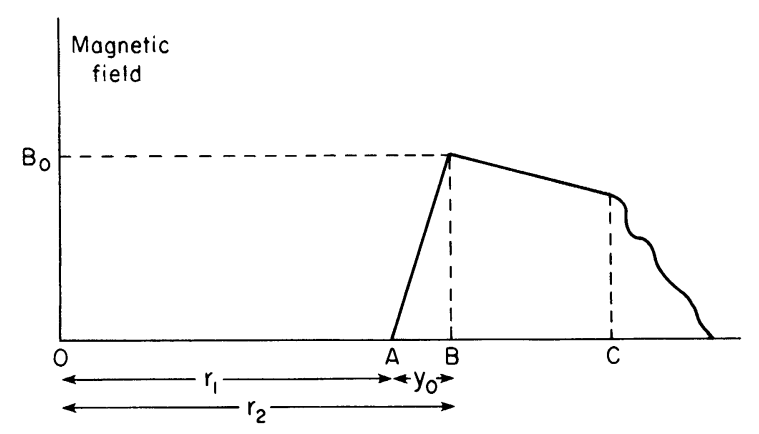

FIGURE 1 Radial distribution of paraxial magnetic field.

\section{MAGNETIC FIELD SHAPING}

A suitable array of currents arranged above and below the ring field region can produce a field pattern of any desired shape in the region $\mathrm{BC}$. If the field is specified at $n$ points in the region BC and at the axis of the system, it can be produced, in principle, by $n+1$ current pairs above and below the axis. Figure 2 shows a pattern produced by specifying the field at three points in the region $\mathrm{BC}$ and requiring it to be zero at the axis. This pattern is produced by currents $I, I$, $-1.6 I$, and $-0.8 I$ at the eight points indicated. Small changes in these currents can yield the pattern shown in Fig. 3. The central field in Fig. 2 is weakly defocusing while that in Fig. 3 is weakly focusing. Either effect will be unimportant compared with the effects of the ring field.

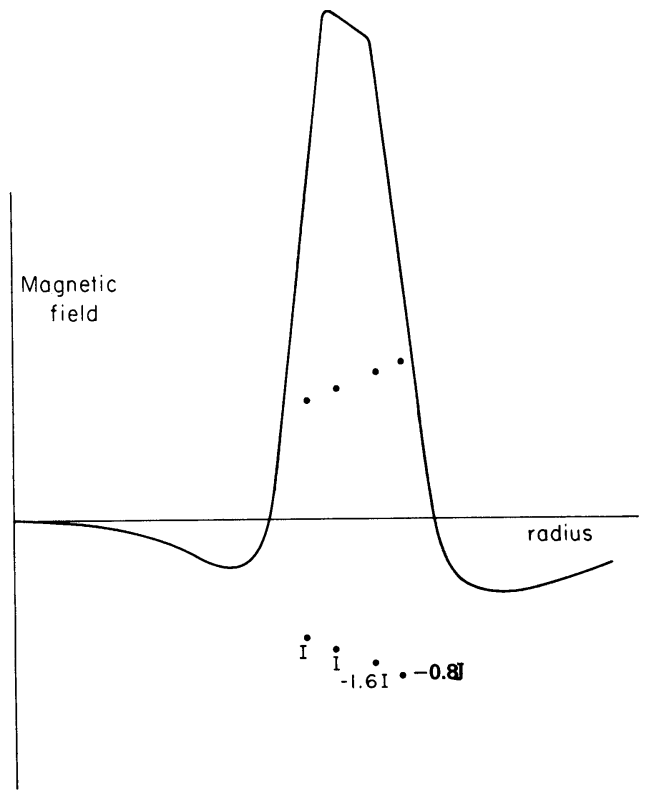

FIGURE 2 Field pattern achievable with eight currents. 


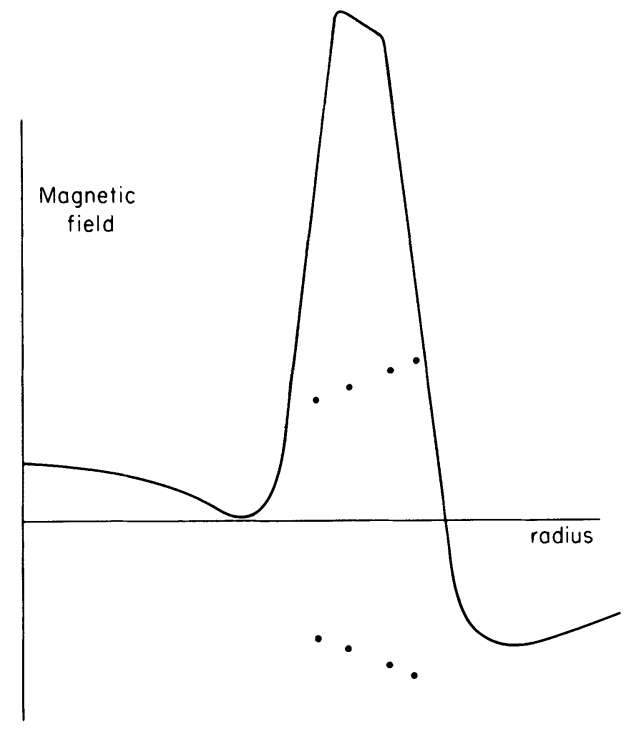

FIGURE 3 Alternative field pattern achievable with eight currents.

The distance $\mathrm{AB}$ (Fig. 1) required for buildup from zero to full field will be about the same as the distance from the median plane to the exciting windings, and so will be about half of the vertical aperture. This will be about the minimum achievable length of $\mathrm{AB}$; it can be increased if, for any reason, this seems desirable.

The current array generating the field will have radial extent considerably greater than BC and will extend both inside of B and outside of C. Since orbits will not extend past $\mathrm{C}$, currents can be included nearer to the median plane at radii greater than $O C$.

\section{ORBITS IN THE RISING FIELD AB}

For radii large compared with the ring's radial extent, the equations of motion boil down to those for rectangular coordinates:

$$
\left.\begin{array}{l}
m \tilde{x}=e \dot{y} B_{z}-e \dot{z} B_{y} \\
m \tilde{y}=-e \dot{x} B_{z} \\
m \ddot{z}=e \dot{x} B_{y}
\end{array}\right\}
$$

Here $\dot{x}$ represents azimuthal motion, $\dot{y}$ represents radial motion and $\dot{z}$ represents motion normal to the median plane (paraxial motion). The constant $e$ is the ionic charge $\left(1.602 \times 10^{-19} \mathrm{C}\right)$, and $m$ is the mass of a proton $\left(1.673 \times 10^{-27} \mathrm{~kg}\right)$ or a deuteron $\left(3.343 \times 10^{-27} \mathrm{~kg}\right)$.

The magnetic field will have two components, $B_{y}$ and $B_{z}$. Because the system is cylindrically symmetric, there will be no B component in the azimuthal direction.

In the range $A B$ of Fig. 1 we represent the axial field by

$$
B_{z}=B_{0}\left(y / y_{0}\right)
$$


where $y$ represents distance from $\mathrm{A}, y_{0}$ is the distance $\mathrm{AB}$, and $B_{0}$ is the field at B. ( $B_{z}$ will be a weak function of $z$; this fact will be neglected in this treatment.) It follows, to the same order of accuracy, that

$$
B_{y}=B_{0}\left(z / y_{0}\right)
$$

We proceed to a solution for the orbit in the median plane. As initial conditions we assume that, at $t=0$, we shall have $x=y=\dot{x}=0$ and $\dot{y}=v_{0}$.

From Eq. (1) it is easily shown that

and

$$
\left.\begin{array}{l}
\dot{x}=\omega y^{2} /\left(2 y_{0}\right), \quad \text { where } \omega=e B_{0} / m, \\
\frac{d^{2}\left(y / y_{0}\right)}{d t^{2}}=-\left(\omega^{2} / 2\right)\left(y / y_{0}\right)^{3} .
\end{array}\right\}
$$

The solution is

$$
y=v_{0} t-\frac{\omega^{2} v_{0}^{3} t^{5}}{4 y_{0}^{2}}+\text { terms in higher powers of } t
$$

For most cases of interest the higher-order terms are negligible for $y<y_{0}$ we can write:

$$
\left.\begin{array}{l}
y=v_{0} t \\
\dot{x}=\omega v_{0}^{2} t^{2} / 2 y_{0} \\
x=\omega v_{0}^{2} t^{3} / 6 y_{0} .
\end{array}\right\}
$$

At $\mathrm{B}$ the entry conditions for the region $\mathrm{BC}$ will be:

$$
\left.\begin{array}{rl}
\dot{y} & =v_{0} \\
\dot{x} & =\omega y_{0} / 2=v_{1} \quad\left(\text { definition of } v_{1}\right) \\
x & =\omega y_{0}^{2} / 6 v_{0} .
\end{array}\right\}
$$

\section{PARAXIAL MOTION BETWEEN A AND B}

To evaluate paraxial motions we assume that excursions from the median plane are not large and that we can superpose the paraxial motion upon the median-plane orbits derived in the preceding section.

From Eqs. (1), (3), and (6), we obtain

$$
\tilde{z}=(e / m) \dot{x} B_{y}=\omega^{2} v_{0}^{2} t^{2} z /\left(2 y_{0}\right)^{2} .
$$

To evaluate the ring magnetic field as a lens, we shall eventually locate the "focal point" on the median plane through which a reflected ion passes after entering the lens parallel to the median plane but with a displacement in $z$. Hence the initial conditions to be applied in the solution of Eq. (8) are $z=z_{0}$ and $\dot{z}=0$. 
The solution is:

$$
\left.\begin{array}{c}
z=z_{0}\left(1+\frac{1}{24}\left(\omega v_{0} / y_{0}\right)^{2} t^{4}+\frac{1}{2688}\left(\omega v_{0} / y_{0}\right)^{4} t^{8}\right. \\
\text { plus higher order terms in } \left.t^{12}, \text { etc. }\right) \\
\left.\dot{z}=\left(z_{0} / 6\right)\left(\omega v_{0} / y_{0}\right)^{2} t^{3}\left(1+\frac{1}{56}\left(\omega v_{0} / y_{0}\right)^{2} t^{4}+\cdots\right)\right)
\end{array}\right\}
$$

At $y=y_{0}, t=y_{0} / v_{0}$ and the terms in the $z$ series are in multiples of $\left(\omega y_{0} / v_{0}\right)^{2}$. This quantity will usually be on the order of unity, so the $t^{8}$ and higher terms can be neglected.

At $B$, the entry conditions for the region $\mathrm{BC}$ will be:

$$
\left.\begin{array}{l}
z=z_{1}=z_{0}\left(1+\frac{1}{24}\left(\omega y_{0} / v_{0}\right)^{2}\right) \\
\dot{z}=u_{1}=\left(\omega^{2} y_{0} z_{0} / 6 v_{0}\right)\left(1+\frac{1}{56}\left(\omega y_{0} / v_{0}\right)^{2}\right) .
\end{array}\right\}
$$

\section{ORBITS IN THE REGION BC}

Between $\mathrm{B}$ and $\mathrm{C}$ the magnetic field can be represented by

$$
B_{z}=B_{0}(1-a y) \text {, }
$$

where $y$ now represents distance from $\mathrm{B}$,

$$
B_{y}=-a B_{0} z \text {. }
$$

Here $a$, which equals $=-\frac{1}{B_{0}} \frac{d B_{z}}{d y}$, is a measure of the field gradient. We expect that desirable values for a will be of the order of 0.1 to 0.2 , i.e., field gradients of $10-20 \%$ per meter.

The equations of motion in the median plane are

$$
\begin{gathered}
\tilde{x}=\omega \dot{y}(1-a y) \\
\tilde{y}=-\omega \dot{x}(1-a y),
\end{gathered}
$$

where $\omega=e B_{0} / m$ as before.

From Eq. (13) we obtain

$$
\dot{x}=v_{1}+\omega y(1-a y / 2),
$$

where $v_{1}$ is the value of $\dot{x}$ at $y=0$ (see Eq. (7)).

The value of $\dot{y}$ follows from

$$
\dot{y}^{2}=v_{0}^{2}-\dot{x}^{2} .
$$

At the maximum excursion into $\mathrm{BC}, \dot{y}$ will drop to zero and $\dot{x}$ will become equal 
to $v_{0}$. Hence, from Eq. (15), $y_{1}$, the maximum value of $y$, is given by

$$
\begin{aligned}
y_{1} & =\frac{1}{a}\left(1-\sqrt{1-2 a\left(v_{0}-v_{1}\right) / \omega}\right) \\
& =\frac{v_{0}-v_{1}}{\omega}\left\{1+\frac{a}{2}\left(\frac{v_{0}-v_{1}}{\omega}\right)+\frac{a^{2}}{2}\left(\frac{v_{0}-v_{1}}{}\right)^{2}+\cdots\right\}
\end{aligned}
$$

The solution to Eqs. (13) and (14) is

$$
\begin{aligned}
& \dot{x}=v_{0} \sin \omega\left(t-a \int y d t\right) \\
& \dot{y}=v_{0} \cos \omega\left(t-a \int y d t\right) .
\end{aligned}
$$

Evidently, so long as $a$ is relatively small, the motion is roughly sinusoidal in time. Detailed analysis shows that Eq. (18) includes a constant term $\left(a v_{0}^{2} / 2 \omega\right)$ which represents the azimuthal precession of the orbit.

For simplicity in studying the effect of the orbital motion on the paraxial motion we rewrite Eqs. (18) and (19) neglecting the $a$ contribution. We assume a time origin that allows us to impose the initial conditions $x=y=0$ and $\dot{x}=v_{1}$. Then

$$
\left.\begin{array}{l}
\dot{x}=v_{0} \sin \omega\left(t+t_{0}\right), \\
\dot{y}=v_{0} \cos \omega\left(t+t_{0}\right), \\
v_{0} \sin \omega t_{0}=v_{1} .
\end{array}\right\}
$$

The orbit returns to $y=0$ when $t=\frac{\pi}{\omega}-2 t_{0}$. At this point,

$$
\begin{aligned}
& \dot{x}=v_{1} \\
& \dot{y}=-\sqrt{v_{0}^{2}-v_{1}^{2}} \cong-v_{0} .
\end{aligned}
$$

\section{PARAXIAL MOTION IN THE REGION BC}

Motion in the $z$ direction must satisfy

$$
\check{z}=\omega \dot{x} B_{y} / B_{0} .
$$

From Eqs. (12) and (20), we find

$$
\check{z}=-\omega a v_{0} z \sin \omega\left(t+t_{0}\right) .
$$

The solution to this equation is

$$
\begin{aligned}
z= & z_{1}\left\{1-\frac{a v_{0}}{\omega}\left[\omega t \cos \omega t_{0}+\sin \omega t_{0}-\sin \omega\left(t+t_{0}\right)\right]\right\} \\
& +\frac{u_{1}}{\omega}\left\{\omega t-\frac{a v_{0}}{\omega}\left[2 \cos \omega t_{0}-2 \cos \omega\left(t+t_{0}\right)-\omega t\left(\sin \omega t_{0}-\sin \omega\left(t+t_{0}\right)\right)\right]\right\}
\end{aligned}
$$




$$
\begin{aligned}
\dot{z}= & a v_{0} z_{0}\left\{\cos \omega\left(t+t_{0}\right)-\cos \omega t_{0}\right\} \\
& +u_{1}\left\{1-\frac{a v_{0}}{\omega}\left[\sin \omega\left(t+t_{0}\right)-\sin \omega t_{0}-\omega t \cos \omega\left(t+t_{0}\right)\right]\right\} \cdots
\end{aligned}
$$

(In these solutions we have neglected certain terms, including $\left(a v_{0} / \omega\right)^{2}$ and higher powers thereof.)

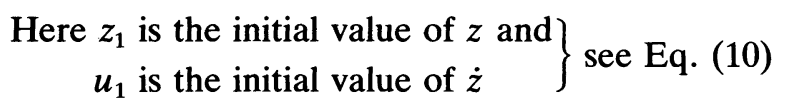

As noted in the previous section, the ion will return to $y=0$ at a time given by $\omega t=\pi-2 \omega t_{0}$. Taking into account that $v_{1} / v_{0}, v_{1} a / \omega$, and $\omega t_{0} / \pi$ all are smaller compared with 1 , and that we are neglecting higher powers of $a v_{0} / \omega$, Eqs. (25) and (26) yield for final values as the ion leaves the region $\mathrm{BC}$ :

$$
\begin{aligned}
z= & z_{2}=z_{1}\left(1-\frac{a v_{0}}{\omega}\left(\pi-2 \omega t_{0}\right) \cos \omega t_{0}\right) \\
& +\frac{u_{1}}{\omega}\left(\pi-2 \omega t_{0}-\frac{4 a v_{0}}{\omega} \cos \omega t_{0}\right) \\
\dot{z}= & u_{2}=-2 a v_{0} z_{1} \cos \omega t_{0} \\
& +u_{1}\left(1-\frac{a v_{0}}{\omega}\left(\pi-2 \omega t_{0}\right) \cos \omega t_{0}\right)
\end{aligned}
$$

\section{ORBIT IN THE REGION BA}

It is reasonably evident that the median-plane return orbit through the region $A B$ will be a mirror image of the initial passage, since the conditons when the ion returns through point $B$ are mirror images of its state when it entered. The ion will emerge at $\mathrm{A}$ with its original velocity reversed and with zero azimuthal velocity.

\section{PARAXIAL MOTION IN THE REGION BA}

In the region BA the equation to be solved for motion in the $z$ direction is Eq. (8), but this time with both an initial velocity and an initial displacement. The sclution at point $\mathrm{A}$, neglecting higher-order terms, is

$$
\begin{aligned}
& z=z_{3}=z_{2}+y_{0} u_{2} / v_{0} \\
& \dot{z}=u_{3}=u_{2}+\omega^{2} y_{0} z_{2} / 6 v_{0} .
\end{aligned}
$$

\section{SIMPLIFICATION AND SUMMARY OF RESULTS}

Our formulae will be greatly simplified if we assume some correlation between parameters. 
From Section 5, Eq. (17), the radial extent of good field, which we shall call $D$, is approximately equal to $\left(v_{0}-v_{1}\right) / \omega$. So we shall write

$$
v_{0}-v_{1}=\omega D \text {. }
$$

It seems reasonable to assume that the vertical aperture in the ring field will be approximately $D$. Hence, as was pointed out in Section 2, we can assume that

$$
y_{0}=D / 2 \text {. }
$$

Now we can evaluate $v_{1}$ from Eq. (7):

$$
v_{1}=\omega y_{0} / 2=\omega D / 4=\left(v_{0}-v_{1}\right) / 4 .
$$

Then we find that:

$$
\left.\begin{array}{l}
v_{1}=0.2 v_{0} \\
\sin \omega t_{0}=v_{1} / v_{0}=0.2, \\
\omega t_{0}=0.201, \\
\cos \omega t_{0}=0.98,
\end{array}\right\}
$$

and

$$
\omega D=v_{0}-v_{1}=0.8 v_{0} .
$$

Incidentally, Eq. (35) indicates that

$$
\left.\begin{array}{rl}
B_{0}(\text { Teslas }) & =2.5 \beta \gamma / D \text { (meters) for protons } \\
& =5 \beta \gamma / D \text { (meters) for deuterons. }
\end{array}\right\}
$$

Using these abbreviations we can summarize the $z$ motion as follows:

$$
\text { Initially } z=z_{0} \text { and } \quad \dot{z}=0
$$

After traversing $\mathrm{AB}$ (from Eq. (10)):

$$
\left.\begin{array}{l}
z_{1} \cong z_{0} \\
u_{1} \cong \omega z_{0} / 12 .
\end{array}\right\}
$$

Returning to B after traversing BC (from (27) and (28):

$$
\begin{aligned}
& z_{2}=z_{0}(1.23-3.77 a D) \\
& u_{2}=z_{0}(0.083-2.55 a D) .
\end{aligned}
$$

Returning to A after traversing BA (from (29) and (30)):

$$
\begin{aligned}
& z_{3}=z_{0}(1.29-4.79 a D) \\
& u_{3}=z_{0}(0.165-2.80 a D) .
\end{aligned}
$$




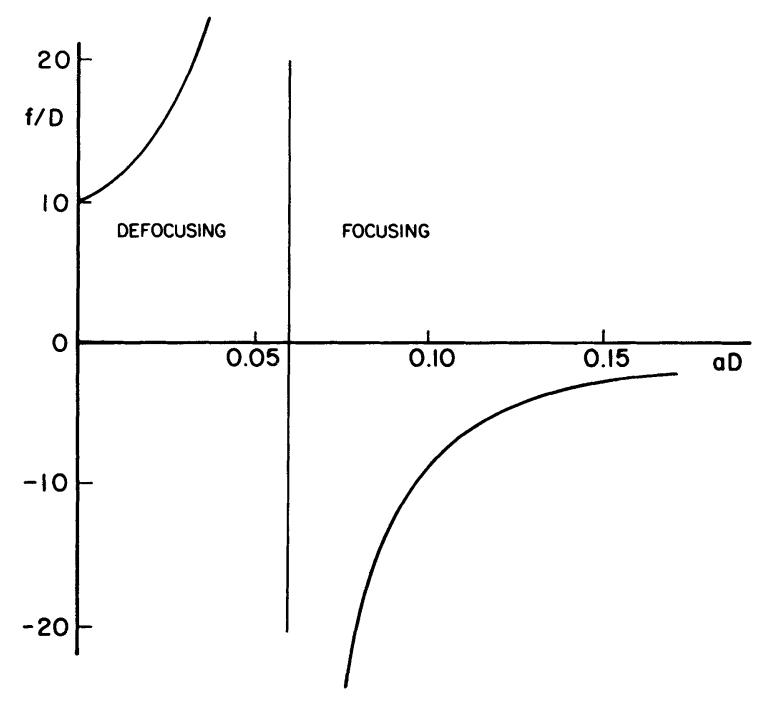

FIGURE 4 Focal length of ring system as a function of total change, $a D$, in magnetic field.

\section{SYSTEM FOCAL LENGTH}

The particle, which entered the "magnetic lens" traveling parallel to the median plane, will cross the median plane at a distance $f$ from point A given by:

$$
f=v_{0} z_{3} / u_{3}=\frac{1.25 D(1.29-4.79 a D)}{0.165-2.80 a D} .
$$

This defines a "focal length" for the system.

Note the important fact that, to this order of approximation, the ratio of $f$, the focal length, to $D$, the depth of the field pattern, depends only on the total fractional field drop $a D$ across the region $\mathrm{BC}$.

In Fig. 4 we show $f / D$ as a function of $a D$. In the region where $a D$ is less than about 0.06 , the focusing strength of the region $B C$ is not enough to overcome the defocusing in $\mathrm{AB}$ and there is net defocusing, i.e., the focal length is positive and the beam emerges as though from a virtual focus outside of $\mathrm{A}$. For $a D$ greater than 0.06 there is net focusing. If, however, $f$ is less than the distance from $\mathbf{A}$ to the axis of the ring, particles will be lost due to "overfocusing." They will reach the opposite ring field with an axial displacement greater than $z_{3}$ and eventually will be lost axially. This phenomenon was first noted by $\mathrm{H}$. J. Berk and $\mathrm{H}$. V. Wong ${ }^{6}$ who point out that it will occur for ions energetic enough to penetrate too far into the focusing part of the ring field.

For example, in a system where the inner radius of the ring field is five times the distance $D$, overfocusing will occur if $a D$ is greater than 0.12 . 


\section{ACCEPTABLE ENERGY SPREAD}

For small values of $a$, the useful radial extent of the bending-focusing field is large; therefore a wide range of energies can be accepted. To establish the order of magnitude of the acceptable energy spread, it was assumed that the upper limit of the energy range is set by $a D=0.12$. A revision of the analysis presented above was carried out to determine the minimum energy, below which defocusing will occur. The minimum energy proved to be less than half of the maximum. Thus it appears, for low values of $a$, that a 2 to 1 energy spread can be accepted.

For large values of $a$, the analysis here presented makes approximations that are no longer valid. As $a$ is increased, it is evident that the extent of the focusing field in the radial direction will decrease and so will the energy acceptance. To a first approximation, it appears that the energy spread will become approximately inversely proportional to $a$ for large values of $a$. In the next section we treat a case where a steeper gradient of field results in a marked decrease in energy acceptance.

\section{SINUSOIDAL FIELD PATTERN}

Orbits have been traced throgh a ring field in whch the paraxial field is

$$
B_{z}=B_{0} \sin \left(\pi y / y_{0}\right) \text {. }
$$

Around the acceptable range, the focal length is given approximately by

$$
f=\frac{y_{0}\left(1.4-0.6 \pi y / y_{0}\right)}{1.85-\pi y / y_{0}}
$$

The limit for defocusing is at $\pi y / y_{0}=1.85$ where $B_{z}=0.96 B_{0}$. The upper limit on

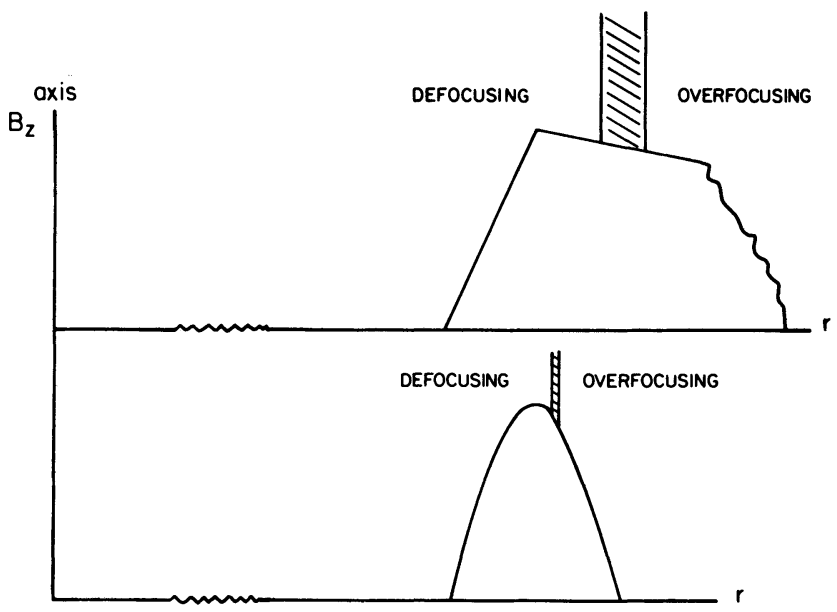

FIGURE 5 Comparison of focusing properties of two field patterns. If the maximum excursion of an ion lies in the shaded area, it will be focused acceptably. 
$y$ for overfocusing in a system where the ring radius is $5 y_{0}$ is given by $f=-5 y_{0}$, which occurs at $\pi y / y_{0}=1.9$ where $B_{z}=0.946 B_{0}$.

In summary, the acceptable range of changes in $B$ is from $4-5.4 \%$. We recall that corresponding figures for the field pattern of Fig. 1 were $6-12 \%$.

In the sine-wave case, the minimum acceptable ion velocity proves to be $96 \%$ of the maximum acceptable velocity. Hence the energy range accepted is about $8 \%$.

In Fig. 5, we indicate the acceptable field ranges for the case of Fig. 1 and for the half-sine-wave case. The acceptable range is shaded in both cases.

\section{CONCLUSION}

For simplicity and clarity in the above treatment, a number of approximations have been made, some fairly drastic. Accordingly, numerical results presented will differ from precise computer determinations of the same parameters. This rough presentation is meant to show the general character of the particle behavior. The final version of Fig. 4 may turn out to have somewhat different scales, but its general shape must be what we have shown.

\section{REFERENCES}

1. R. Macek and B. Maglich, Part. Accel. 1, 2 (1970), p. 121.

2. B. Maglich et al, Phys. Rev. Lett. 27, 14 (1971), p. 909.

3. B. Maglich, Atomkernenergie, 32 (1978), p. 100.

4. B. Maglich, Nucl. Instrum. Meth. A 271, 1 (1988), pp. 13 and 167.

5. J. P. Blewett, Nucl. Instrum. Meth. A 271, 1 (1988), p. 214.

6. H. L. Berk and H. V. Wong, "Overfocusing in a Migma and in Exyder," companion paper in this issue. 